\title{
Forecasting Mortality Convergence up to 2100
}

\author{
Alessandra Garbero (garbero@iiasa.ac.at) \\ Warren Sanderson (wsanderson@notes.cc.sunysb.edu)
}

\section{Approved by}

Wolfgang Lutz

Leader, World Population Program

December 04, 2012

Interim Reports on work of the International Institute for Applied Systems Analysis receive only limited review. Views or opinions expressed herein do not necessarily represent those of the Institute, its National Member Organizations, or other organizations supporting the work. 


\section{Contents}

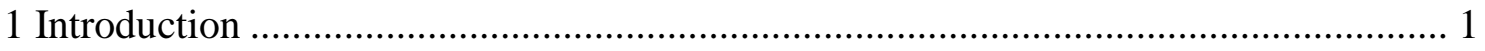

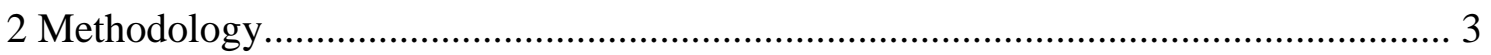

3 Estimation Procedure.................................................................................... 5

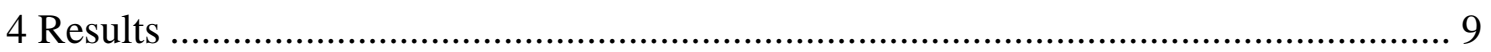

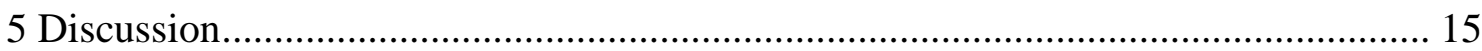

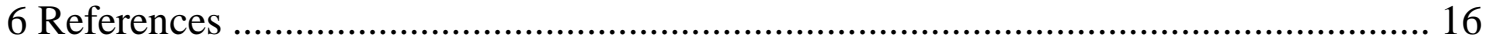




\section{Abstract}

This paper presents life expectancy forecasts for all countries in the world explicitly assuming mortality convergence. We develop a model that takes into account countryspecific heterogeneity in life expectancy historical trajectories, between-countries heterogeneity across gains and uncertainty through experts' based arguments (Lutz et al. 2001). The relevant literature has focused on forecasting mortality for a single population. Exception to this rule is the work by Li and Lee (2005) where the authors develop mortality forecasts that take into account patterns in a larger group using the Lee-Carter model.

Torri and Vaupel (2012) argue that life expectancy in different countries tends to be positively correlated and forecast life expectancies in individual countries by forecasting the best-practice level and the gap between the national performance and the bestpractice level. We build upon their work by varying the speed of convergence, taking into account differential rates of linear increase in life expectancy across group of countries. 


\section{Acknowledgments}

Funding for this work was made possible by the European Research Council (ERC) Advanced Investigator Grant focusing on "Forecasting Societies' Adaptive Capacities to Climate Change” (ERC-2008-AdG 230195-FutureSoc). 


\section{About the Authors}

Alessandra Garbero is an Econometrician working at the International Fund for Agricultural Development (IFAD). She is also a Research Scholar at IIASA's World Population Program.

Warren C. Sanderson is Professor of Economics and Professor of History at Stony Brook University in New York. He is also an Associate Research Scholar at IIASA's World Population Program. 


\title{
Forecasting Mortality Convergence up to 2100
}

\author{
Alessandra Garbero and Warren Sanderson
}

\section{Introduction}

In summer 2011, a global Internet survey on the likely future trends in fertility, mortality, and migration and the main factors behind them was conducted among the members of major population associations and selected other professional organizations. The survey, a collaboration between the International Institute for Applied Systems Analysis' (IIASA) World Population Program and Oxford University, constitutes a basis for new population forecasts by age, sex, and level of education for most of the countries of the world and the provinces of India and China. By allowing a large number of experts to participate and by providing an "argument-based" underpinning of numerical estimates about future trends in life expectancy in high and low mortality countries, the survey addresses two common weaknesses of population projectionmaking: 1) a very limited or no theoretical foundation and 2) a participation of a small and often closed group of experts formulating the parameters of projection scenarios.

This "argument-based" approach to produce probabilistic population projections has been developed and refined at IIASA over the past decade. Nathan Keyfitz and Andrei Rogers laid out the methodological foundations during the 1970s and early 1980s, and later Wolfgang Lutz translated these concepts into sets of systematic numerical population forecasts for world regions. In general, introducing experts' opinions within population forecasting offers the useful advantage of providing a wealth of information, demographic as well as epidemiological, at least in a qualitative way. A limitation is its potential of being prone to bias, namely experts' subjectivity or their need to conform (for a review see Booth and Tickle 2008).

In order to reduce bias, Lutz and Scherbov (1998) proposed Delphi-methods to draw on a wider expertise, thereby averaging out expert opinions.

In this paper, we put greater emphasis on experts' arguments rather than opinions as in successive developments of the methodology (Lutz et al. 2001; Lutz and Goldstein 2004). Experts' arguments have been shown to increase the range of expectations. Experts' expectations approximate population-level behavior and have been found to be particularly suited for medium to longer term forecasting (Booth and Tickle 2008).

As part of this endeavor to produce a new set of argument-based population forecasts, a number of workshops were organized to validate ex-post the estimates in the survey given by the source experts. Leading experts in the field were asked to comment 
upon and reach consensus about the future of life expectancy in high and low mortality ${ }^{1}$ countries in two meetings, held respectively in Cape Town, South Africa (February 2012) and in San Jose, Costa Rica (February 2012). In the Costa Rica meeting, the majority of experts agreed on a general paradigm of mortality convergence.

The concept of convergence is central to the demographic transition theory and the presence of a global mortality convergence has been largely documented (Wilson 2001). This idea initially arose from analyses that were performed on mortality data starting from the 1960s mostly coming from developed countries (McMichael et al. 2004). In addition, the occurrence of a common pattern, named the "epidemiological transition” (Omran 1971), with the latter term broadly indicating falling death rates from infection diseases (mostly linked to young ages and premature mortality) to increasing deaths from non-communicable disease (largely occurring in old-age), gave some expectation of a rapid decline of mortality in high mortality countries as well, and stagnation for countries which already achieved a relatively low level of mortality.

Although the idea of a global mortality convergence is widely acknowledged (Wilson 2001), there have been relatively few attempts to deal with convergence explicitly (Wilson 2001; Heuveline 1999; Oeppen 2006). On the other hand, the concept of convergence has been largely employed in the economics literature and particularly in the empirical literature on economic growth (Barro and Sala-I-Martin 1995; Landau et al. 1996). In the latter there are two main concepts of convergence; the first one, betaconvergence, occurs when the growth rate of the variable of interest (normally the growth rate in GDP) depends negatively on its prior value. Controlling for the influence of other factors this produces the case of conditional convergence, where the level of convergence depends on those other factors. The second concept is sigma-convergence, which occurs when the dispersion of the indicator decreases. It's just a description, without any assumption on the functional relation, in contrast to the beta-convergence. In the literature (Anand and Ravallion 1993; Bidani and Ravallion 1997) there are different sub-concepts of sigma convergence.

Using the concept of sigma-convergence in absolute terms, this paper produces female life expectancy forecasts for 159 countries in the world.

Specifically we develop a multi-pronged methodology that takes into account country-specific heterogeneity in life expectancy historical trajectories of lifeexpectancies, between-country heterogeneity across life expectancy gains and argument-based expert' judgement (Lutz et al. 1998; Lutz et al. 2001).

The literature on forecasting mortality has usually focused on forecasting mortality for a single population (for a review see Hyndman et al. 2011). Exception to this rule, is the work by $\mathrm{Li}$ and Lee (2005) where the authors argue that mortality trajectories and patterns are increasingly becoming more similar in closely related populations and therefore develop mortality forecasts that take into account patterns in a larger group using the Lee-Carter model (1992). In this paper, we go a step forward and forecast country-specific life expectancy within a regional framework.

\footnotetext{
${ }^{1}$ High and low mortality populations were separated on the basis of the level of child mortality in the year 2010 according to the revised estimates from the Inter-agency Group for Child Mortality Estimation (IGME), with the threshold being 40 deaths per 1,000 children below age 5 .
} 
In addition we take into account existing work by several authors (White 2002; Oeppen and Vaupel 2002; Lee 2003; Torri and Vaupel 2012). The latter have stressed three major points: the first is that life expectancy (record or average) appears to have changed linearly over long periods of time. The second is that "national mortality trends should be viewed in a larger international context rather than being analysed and projected individually" (Lee 2003). The third is that life expectancy in different countries tends to be positively correlated. Torri and Vaupel (2012) forecast life expectancies in individual countries by forecasting the best-practice level and the gap between the national performance and the best-practice level.

We build upon the work by Torri and Vaupel (2012), by explicitly varying the speed of convergence i.e. taking into account differential rates of linear increase in life expectancy across group of countries. Other authors (Raftery et al. 2012; Lalic and Raftery 2011) have already stressed this point and highlighted that life expectancy has been increasing most quickly for countries with middle life expectancy (i.e. around 60 years), and more slowly for countries with lower or higher levels. With respect to these authors, we subdivide countries between forerunners and laggards, propose a one-sex model and generate life expectancy forecasts that are a function of country-specific stochastic and structural trends in life expectancy as well as a function of stochastic and structural differences between forerunners and non-forerunners. Hence, we explicitly take into account different forms of heterogeneity: 1) the heterogeneity among forerunners-specific gains as well as among non-forerunners/laggards' ones; 2) the relationship between the two, and 3) the heterogeneity across countries and regions. The model is explained in detail in the next section.

\section{Methodology}

Our forecasts are based on estimates of female life expectancy from 1950 to 2005 (United Nations 2011).

Specifically, we assume that country-specific life expectancy will converge to the one of the global forerunner by 2100 . The global forerunner has been defined as "the best practice life expectancy" (BPL), a time series that includes Japan as well as other countries that historically exhibited the highest level of life expectancy during the period spanning from 1950 to 2005. The forecast for the global forerunner is based on a linear increase in life expectancy of two years per decade until 2100 (Oeppen and Vaupel 2002; Sanderson and Scherbov 2004).

Although it is plausible to believe that low mortality countries will exhibit linear increases in life expectancy and will converge to an upper bound level of life expectancy by 2100 , the paradigm is less applicable to high mortality countries. To this end, we developed the idea of a two-steps convergence process, where countries were subdivided into regional forerunners and laggards vis a vis the global forerunner. The idea is that laggards' life expectancy will continue to grow as fast as in the regional forerunner. Regional forerunners were defined as countries that exhibited the highest life expectancies in 2005-2010 within each geographical region.

The model is implemented as a dynamic panel data model, autoregressive of order 1, with fixed effects, which is estimated with 2-step GMM. The specification is the following and is estimated over the period 1980-2005: 


$$
\Delta e 0 c_{t, t-1=} \gamma \Delta e 0_{i, t, t-1}+\beta\left(e 0_{i, t-1}-e 0_{c, t-1}\right)+\varepsilon_{c, t}+\epsilon_{c}
$$

Where $e 0_{i, t}$ is the female life expectancy at birth for the forerunner $i$ at time $t$ $e 0_{c, t}$ is the female life expectancy at birth for the country $c$ at time $t$

$\Delta e 0 c_{t, t-1}$ is the change in female life expectancy at birth for the country $c$ between $t$ and $t-1$

$\Delta e 0 i_{t, t-1}$ is the change in female life expectancy at birth for the forerunner $i$ between $\mathrm{t}$ and $t-1$

$\varepsilon_{c, t}$ is the time-varying error component

$\epsilon_{c}$ are country-specific fixed effects.

It is important to note that the equilibrium is reached when:

$$
\left(e 0_{i, t-1}-e 0_{c, t-1}\right)=\frac{(\gamma-1) \Delta e 0_{i t, t-1}+\epsilon_{c}}{\beta}
$$

The "beta term" is endogenous. In order to correct for the dynamic panel bias and auto-correlation, we instrument this term with its fourth and fifth lag. The SarganHansen test indicates that the instruments are valid, and the result from the endogeneity test corroborates the fact that the beta term is endogenous (the latter is defined as the difference of two Sargan-Hansen statistics: one for the equation with the smaller set of instruments, where the suspect regressor are treated as endogenous, and one for the equation with the larger set of instruments, where the suspect regressors are treated as exogenous).

In summary, we are trying to estimate a model where life expectancy gains (or changes between $t$ and $t-1$ ) for each country are regressed against the forerunner life expectancy gains controlling for the gap (the "beta term") between the forerunner life expectancy and the country itself observed in the previous period ( $t-1)$. The lags are computed over the period from 1950 to 2005.

Hence, convergence is achieved when the difference between country-specific life expectancy and the forerunner is no longer changing. In essence, we have a model in which only the mean of distribution of life expectancies changes over time, but the variance remains constant. In addition, our model does not impose an upper limit. There is no convergence to the level of the forerunner, in part because the level is always changing.

The added value of this convergence model lies in the fact that it is based on empirical data. In addition, it takes into account the heterogeneous country-specific historical experience as well as differences in gains between forerunners and laggards over time and across regions. Thus, it takes into account structural as well as stochastic components that contribute to life expectancy trends overtime and it is able to generate unbiased parameters upon which the new forecasts are based. 


\section{Estimation Procedure}

The estimation procedure encompasses five steps.

The first step involved generating the forerunners life expectancy forecasts (2010-2100).

The second step involved generating life expectancy forecasts for the nonforerunners. Separate regressions were conducted for Southern and South-East Asia to obtain region-specific parameters (2010-2100).

The third step involved generating life expectancy forecasts for HIV-affected countries as well as high mortality countries such as Afghanistan (2050-2100).

The forth step involved incorporating source experts and meta-experts opinions in the form of net gains in life expectancy, from the low and high mortality surveys (2020-2050).

The final step consisted of recalibrating the future trajectories (2050-2095) based on new trend and estimated parameters, since experts opinions were only given until 2050.

\section{Step 1: Generating forerunners life expectancy forecasts}

In order to generate the forerunners life expectancy forecasts, we estimated the model using as the global forerunner, a "best-practice" life expectancy (BPL) which includes Japan as well as other countries that historically exhibited the highest life expectancies during the period spanning from 1950 to $2005^{2}$. The estimation sample for the first stage regressions included the following countries:

\footnotetext{
${ }^{2}$ Specifically: Norway (1950-55/1955-60); Iceland (1960-65), Sweden (1965-70/1970-75); Iceland (1975-80); Japan (1980-85 to 2005-10).
} 
Table 1. Estimation sample

\begin{tabular}{|c|c|}
\hline Region & Country \\
\hline Oceania & Australia \\
\hline Caribbean & Martinique \\
\hline Central America & Costa Rica \\
\hline Central Asia & Kazakhstan \\
\hline Eastern Africa & Reunion; Mauritius; Mayotte; Madagascar \\
\hline Eastern Asia & Japan \\
\hline Eastern Europe & Czech Republic \\
\hline Melanesia & New Caledonia \\
\hline Micronesia & Guam \\
\hline Middle Africa & Sao Tome and Principe; Gabon \\
\hline NORTHERN AMERICA & Canada \\
\hline Northern Africa & Libyan Arab Jamahiriya; Tunisia \\
\hline Northern Europe & Iceland \\
\hline Polynesia & French Polynesia \\
\hline South America & Chile \\
\hline South-Eastern Asia & Singapore \\
\hline Southern Africa & Namibia \\
\hline Southern Asia & Sri Lanka \\
\hline Southern Europe & Italy \\
\hline Western Africa & Cape Verde; Ghana \\
\hline Western Asia & Israel \\
\hline Western Europe & France \\
\hline
\end{tabular}

This model fits the data well and succeeds in capturing historical converging trends across this set of countries. Exceptions are however some African countries (Madagascar and Ghana - while the fit for Namibia and Gabon is largely acceptable and surprisingly converging towards the UN forecast value in 2100). Given the presence of HIV and AIDS, we don't assume convergence for the period 2010 to 2050 for HIV and AIDS affected countries as well as high mortality countries such as Haiti and Afghanistan. The latter are exceptions to the convergence theory, due to the occurrence of mortality reversals over the last two decades. Factors such as conflicts, failure of health systems and impact of HIV and AIDS epidemic have largely impeded progress in life expectancy in the past 20 years and will probably continue to impede it in the short term. For this subset of countries, the United Nations medium variant was adopted up to $2050^{3}$ (United Nations 2010). Therefore we deliberately endorse the UN medium

\footnotetext{
${ }^{3}$ Although India is considered an HIV and AIDS affected country in the UN medium variant scenario, we decided to explicitly model convergence for India as well, given the low prevalence of HIV.
} 
scenario as the correct forecast, given the impact of mortality crisis which the model cannot capture.

Therefore, based on the parameters obtained from this model, we produced forecasts for the regional leaders (Japan, Tunisia, Sri Lanka, French Polynesia, Guam, New Caledonia, Singapore, Costa Rica, Martinique, Kazakhstan, Israel, Reunion and Madagascar). As Madagascar represents an outlier in terms of its past mortality experience (Ranis and Stewart 2000), we imposed an average parameter which summarizes the historical experience of the African forerunners (i.e. Tunisia, Reunion, Mauritius, Gabon, Ghana, Sao Tome and Principe and Cape Verde).

Table 2. Forerunners for second-stage regression

\begin{tabular}{|l|}
\hline Europe \& North America: Japan \\
\hline Northern Africa (including Sudan): Tunisia \\
\hline Eastern Africa: Madagascar \\
\hline Western Africa: Ghana \\
\hline Middle Africa: Gabon \\
\hline Southern Africa: Namibia \\
\hline Southern Asia: Sri Lanka \\
\hline Polynesia: French Polynesia \\
\hline Micronesia: Guam \\
\hline Melanesia: New Caledonia \\
\hline South-Eastern Asia: Singapore \\
\hline South America/Central America: Costa Rica \\
\hline Caribbean: Martinique \\
\hline Western Asia: Israel \\
\hline Central Asia: Kazakhstan \\
\hline Hybrid group (including Cape Verde, Comoros, Mauritius, Mayotte, Sao Tome and Principe): Reunion \\
\hline
\end{tabular}

\section{Step 2: Generating non-forerunners life expectancy forecasts}

The second step involved running the model on all the remaining countries. We stratified the model by region i.e. Southern Asia, South-East Asia and the remaining countries (excluding countries that belong to the HIV-affected group as well as Haiti and Afghanistan). This time the regional forerunners life expectancies were chosen as "best-practice" life expectancies for each region. Based on the parameters obtained from the second stage regression, we generated forecasts up to 2100 .

\section{Step 3: Generating non-forerunners life expectancy forecasts (HIV and AIDS affected countries and Afghanistan}

The third step involved generating life expectancy forecasts from 2050 to 2100, for HIV-affected countries and high mortality countries such as Haiti and Afghanistan. We explicitly assume convergence for this projection interval. Namibia was chosen as a forerunner for this set of countries for three reasons. First, its high life expectancy 
across the period 2005-2010 in the entire Sub-Saharan Africa region (United Nations 2011); second, for its high levels of anti-retroviral treatment provision in 2010 to the large percentage of affected population (UNAIDS 2010); third, for its large investments in the health sector (GHI 2011). We retrieved the structural parameter that is implicit from the first step regression and imposed it for the period 2050-2100. This parameter embodies all unobserved characteristics (for instance success of health care system, economic growth, education expansion) that characterize life expectancy gains in Namibia. By retrieving this parameter and using the Namibian forecast as well the parameters beta and gamma from the second stage regression we generated the structural parameters for the countries falling in this group and produced our forecasts from 2050-2100. Remarkably these forecasts are quite close to the UN ones for the period 2050-2100.

\section{approach}

Step 4: Incorporating uncertainty through an experts' based argument

The fourth and quite important step involved the reweighting of these forecasts in order to incorporate source experts and meta-experts arguments in the form of life expectancy net gains. Through this step, we aim at incorporating uncertainty in our forecasts based on an expert-based argument methodology (Lutz et al. 2001).

In order to take into account source experts (i.e. experts that answered the survey) and meta-experts (i.e. experts that attended the workshops) numerical estimates (decadal gains in life expectancy), we implemented a framework that operationalizes source-experts and meta-experts arguments and combines their estimates with our life expectancy forecasts.

Analytically, given model gains $e 0_{m c, t}$, which consist of the model gains implied by our forecasts for all countries except Sub-Saharan Africa, Haiti and Afghanistan, and the ones implied by UN medium scenario for the other set of countries, we reweighted model gains (from 2010-2050) to obtain new model gains $e n_{0 m}$ :

en $_{0 m}=1 * e 0_{m c, t}+0.2 \quad *$ IndSourceExpGains $_{c, t}+1 *$ AvgMetaExpGain $\left._{c, t}\right) /$ $\sum$ Weights

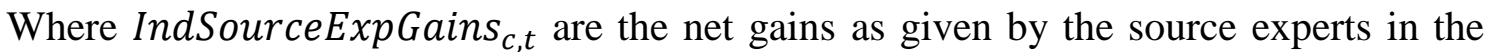
low and high mortality surveys. The latter were given a weight of 0.2 .

AvgMetaExpGain, is the average of the net gains as given by the meta-experts ${ }^{4}$ in the low and high mortality surveys. The latter are given a weight of 1 .

\footnotetext{
4 One additional complication was that the metric was different (gains versus life expectancy point estimates) as meta-experts gave only predicted life expectancy for both sexes for two point estimates (i.e. 2030 and 2050) for high mortality countries. Therefore, we calculated the e0 differential (female life expectancy - total) in 2005 and derived predicted female life expectancy (assuming that this difference remains constant to the 2005 level). We then performed quadratic interpolation to reconstruct "missing" net gains. In addition, we kept the model gains until 2015 for HIV and AIDS affected countries and then interpolated between 2015 and first available gain (2020) given by the experts - hence, there is difference in the magnitude of gains between our predicted gains and the ones implied by the UN medium scenario until 2050.
} 
Also model gains $e 0_{m c, t}$ are given a weight of 1 . The magnitude of these weights was assessed through a sensitivity analysis.

The new model gains were then employed to adjust the model forecasts up to 2050.

\section{Step 5: Generating forecasts for all countries for the period 2050-2100}

The final step consisted of modifying the future trajectories (2050-2095) based on the new trend implied by the reweighted forecasts up to 2050. We computed the structural parameter (fixed effect) which is implied by the new forecast (2010-2050) and combined with the parameters (beta and gamma) that have been estimated throughout the various phases of our procedure to obtain forecasts for the period 2050-2100.

\section{Results}

We present results after the first step for the forerunners (Figure 1) and the overall procedure for the United States of America, Brazil, Botswana, Tajikistan, Ethiopia, China and Ghana (Figure 2 to Figure 8). The final set of graphs (Figures 2 to 8) present our final forecast (final) i.e. after step 5, the model value obtained after step 3 (model), the UN life expectancy value $(U N)$, and the forerunner life expectancy $(B P L)$. For countries with no experts' numerical estimates ${ }^{5}$, the procedure stops after the third step (i.e. model values coincide with our final forecast value) hence the resulting three lines in the figures.

Figure 1 shows the forerunners forecasts after the first step of the procedure. For HIV and AIDS affected countries this first step is only illustrative of how the model fits the data and how the forecasts would look, had we employed the parameters to generate the forecasts. For instance, the final forecast for Ghana is presented in Figure 8 after step 5 is implemented (i.e. reweighting due to experts' predicted decadal gains and calibration of trend after 2050).

Figure 2 (United States) shows how historically the United States diverged from Japan. However our model is generally optimistic in generating linear gains and a parallel line. For this country, we had the largest number of experts in the low mortality survey. The latter provided a distribution of decadal gains in life expectancy for 202030 and 2040-50. After adjusting the model forecast taking into account experts' numerical estimates, the forecast is adjusted downward indicating their pessimistic view.

In Brazil, Figure 3, experts had provided a more optimistic outlook about the future of life expectancy, which results in an adjustment upward of our model forecast.

As far as Botswana, Ghana and Ethiopia were concerned, experts were largely pessimistic (resulting in an adjustment downward of the line). However our model results did not exclude a possible catch up in the period 2050-2100.

In general, our forecasts are largely optimistic when compared to the United Nations' ones. Exceptions are HIV and AIDS affected countries, Sub-Saharan Africa as well as high mortality countries such as Afghanistan. In these instances, our final forecast values are either lower, or in substantial agreement (i.e. Afghanistan and Haiti).

\footnotetext{
${ }^{5}$ The low and high mortality surveys provided data only for 30 and 14 countries respectively.
} 

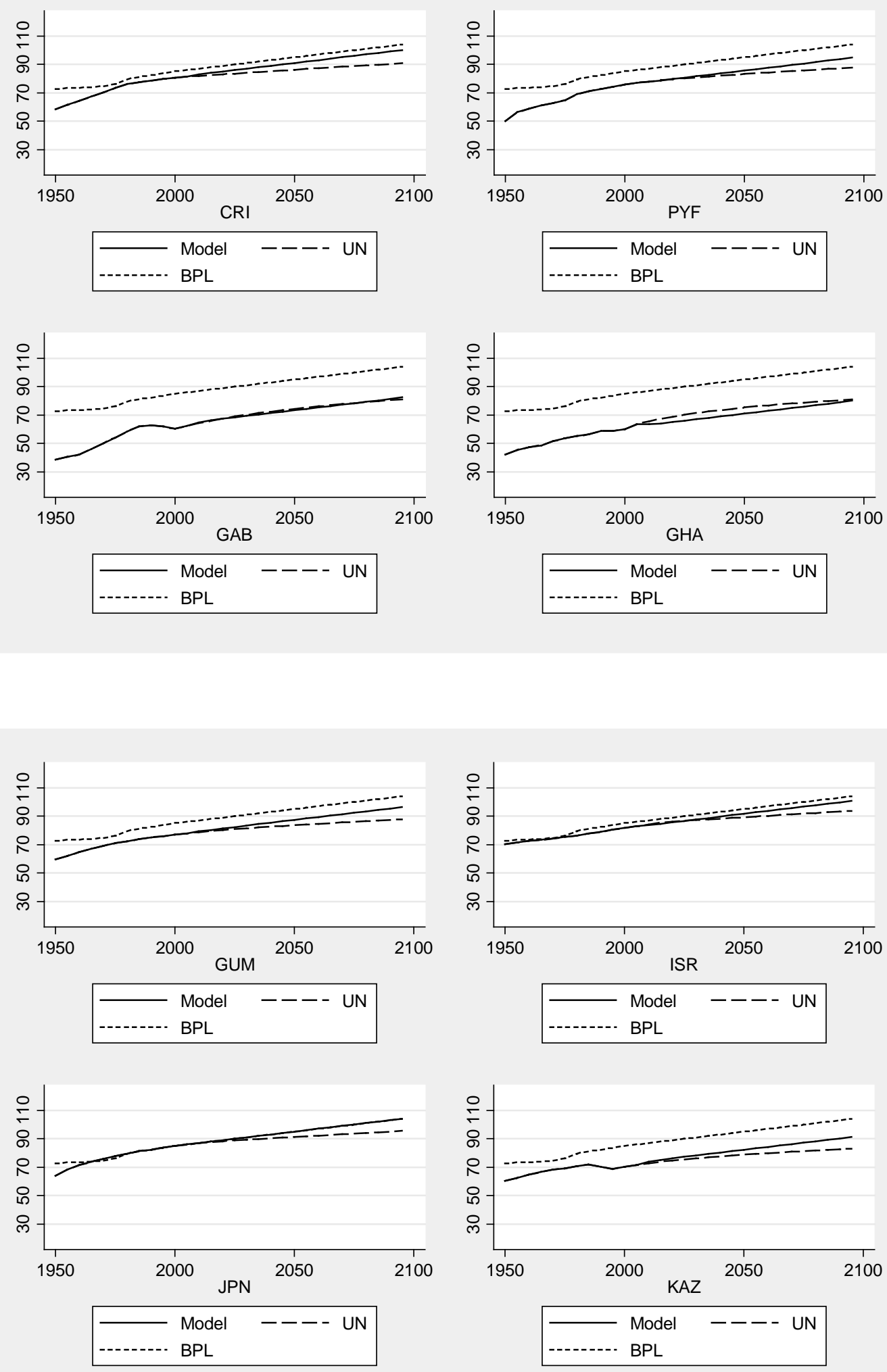

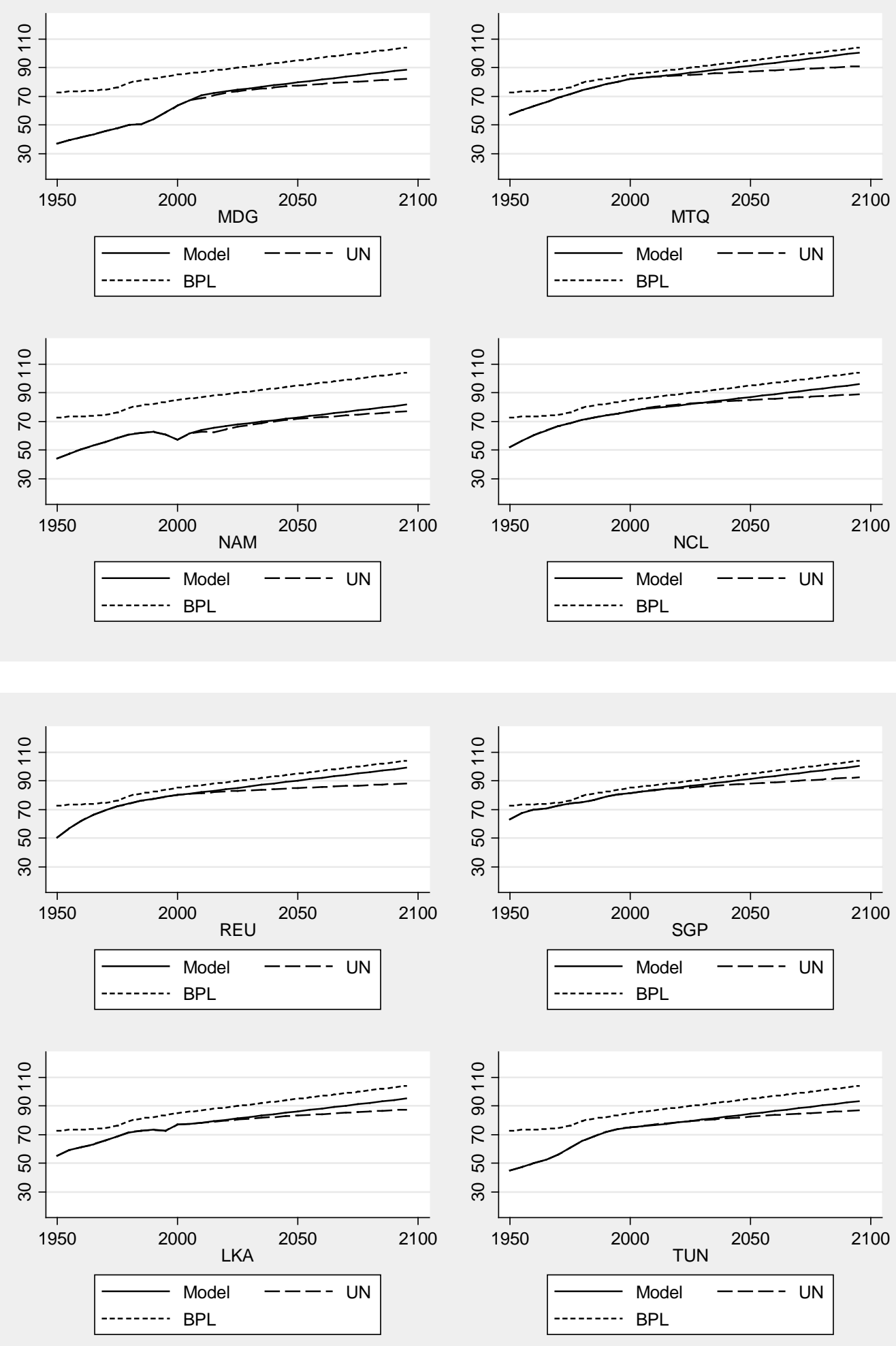

Figures 1a-d. Step 1 - Forerunners forecasts (Model forecast, UN medium scenario vs. global forerunner $\mathrm{e} 0$-BPL) 


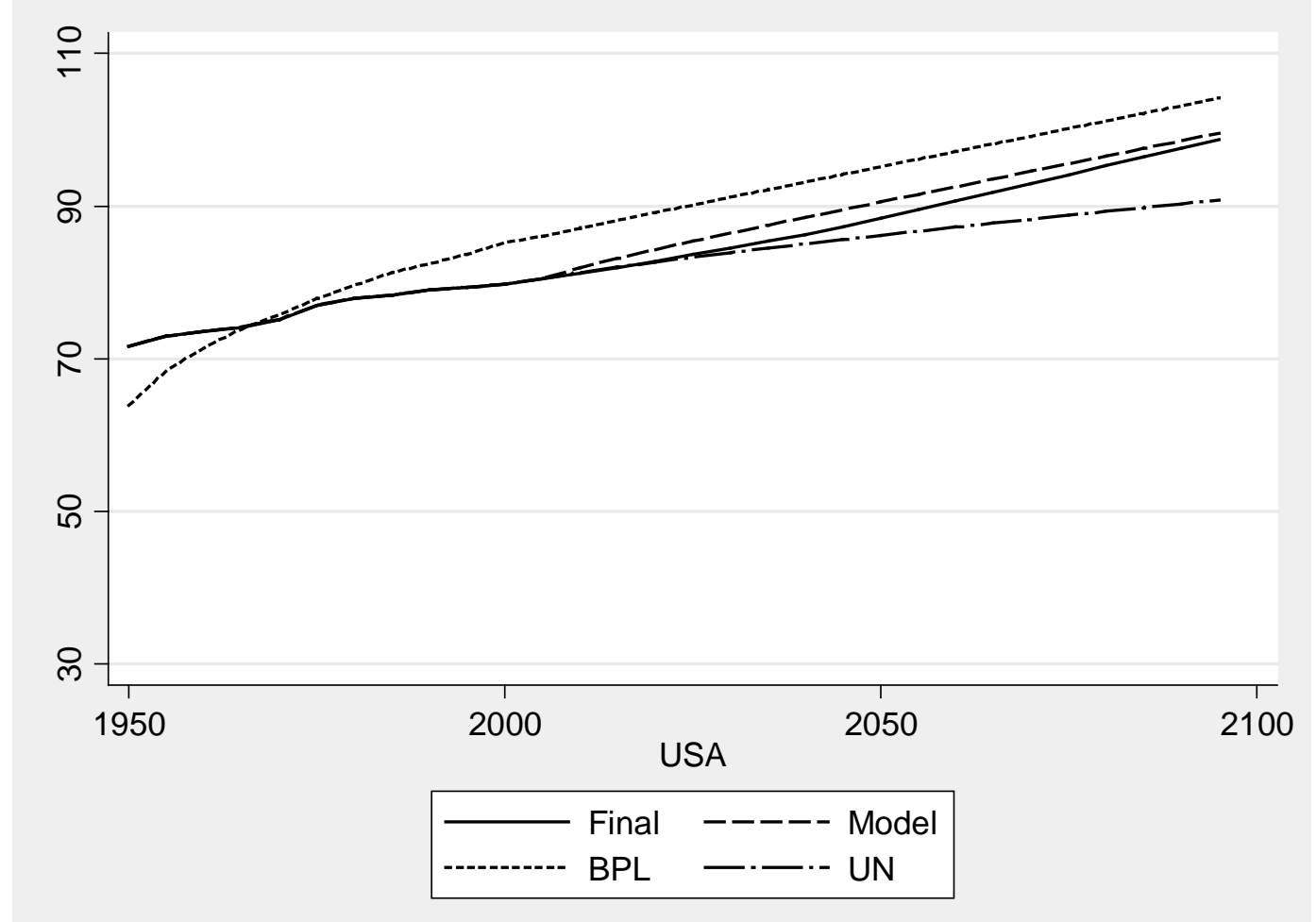

Figure 2. United States of America (BPL: Japan)

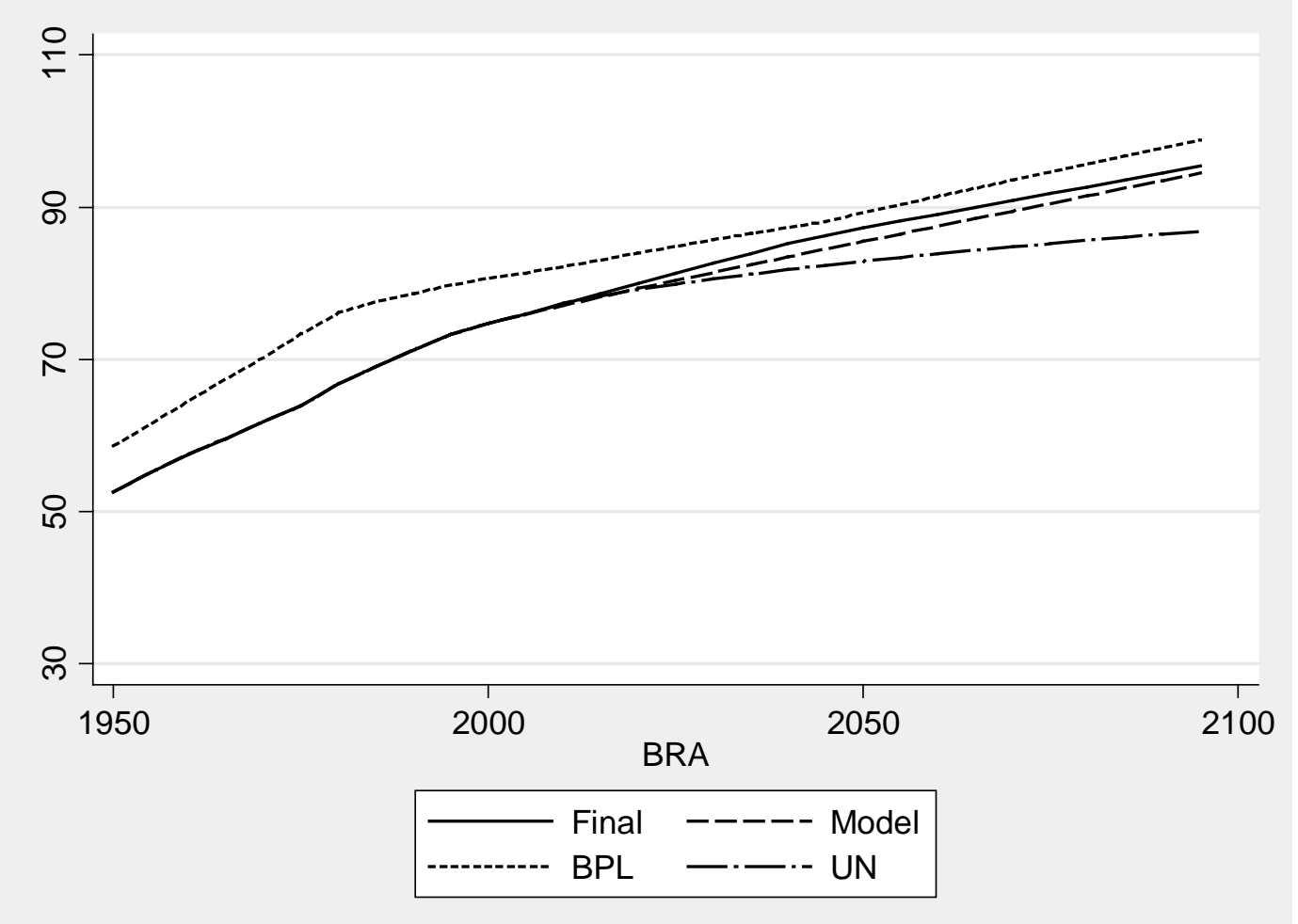

Figure 3. Brazil (BPL: Costa Rica) 


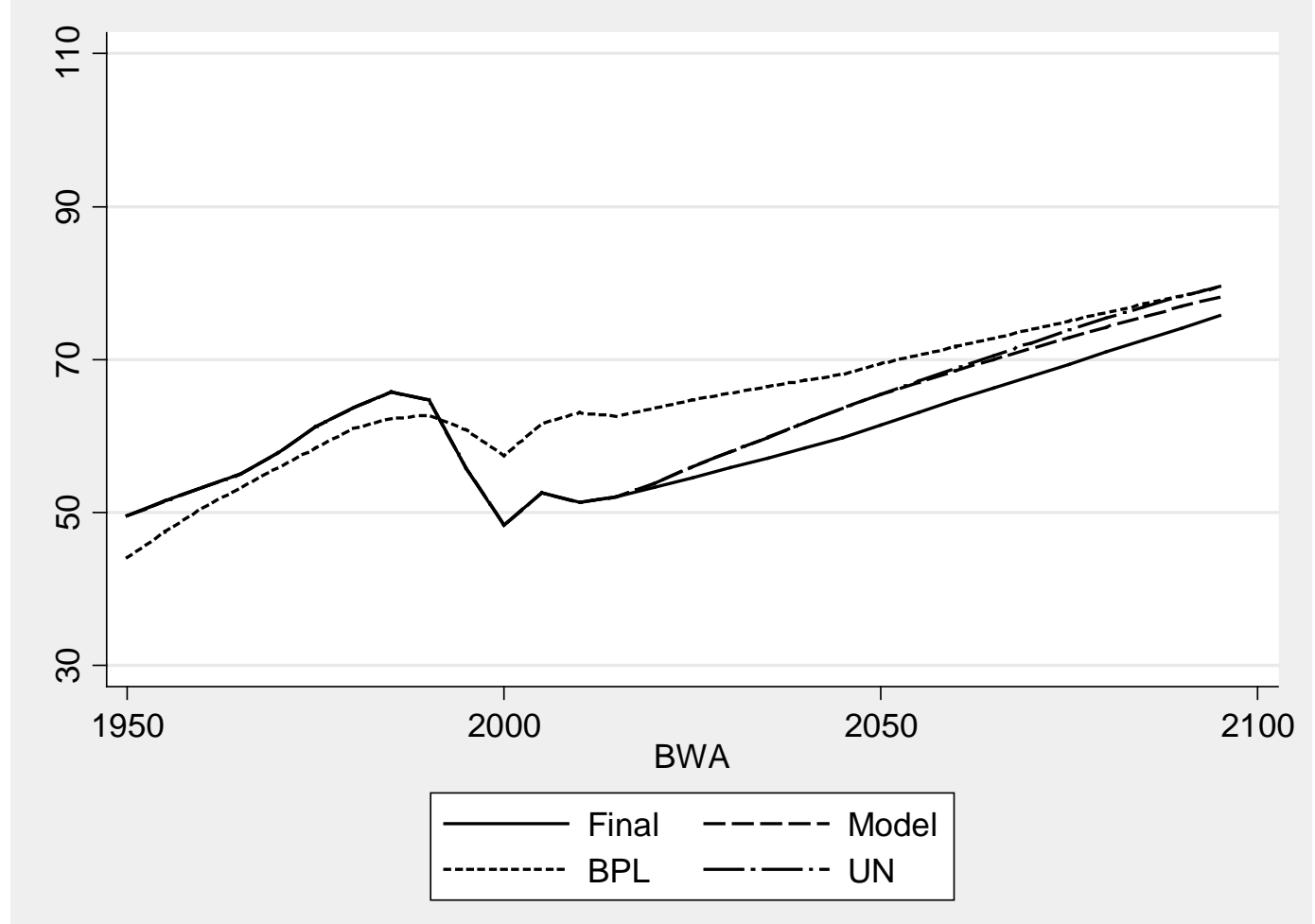

Figure 4. Botswana (BPL: Namibia)

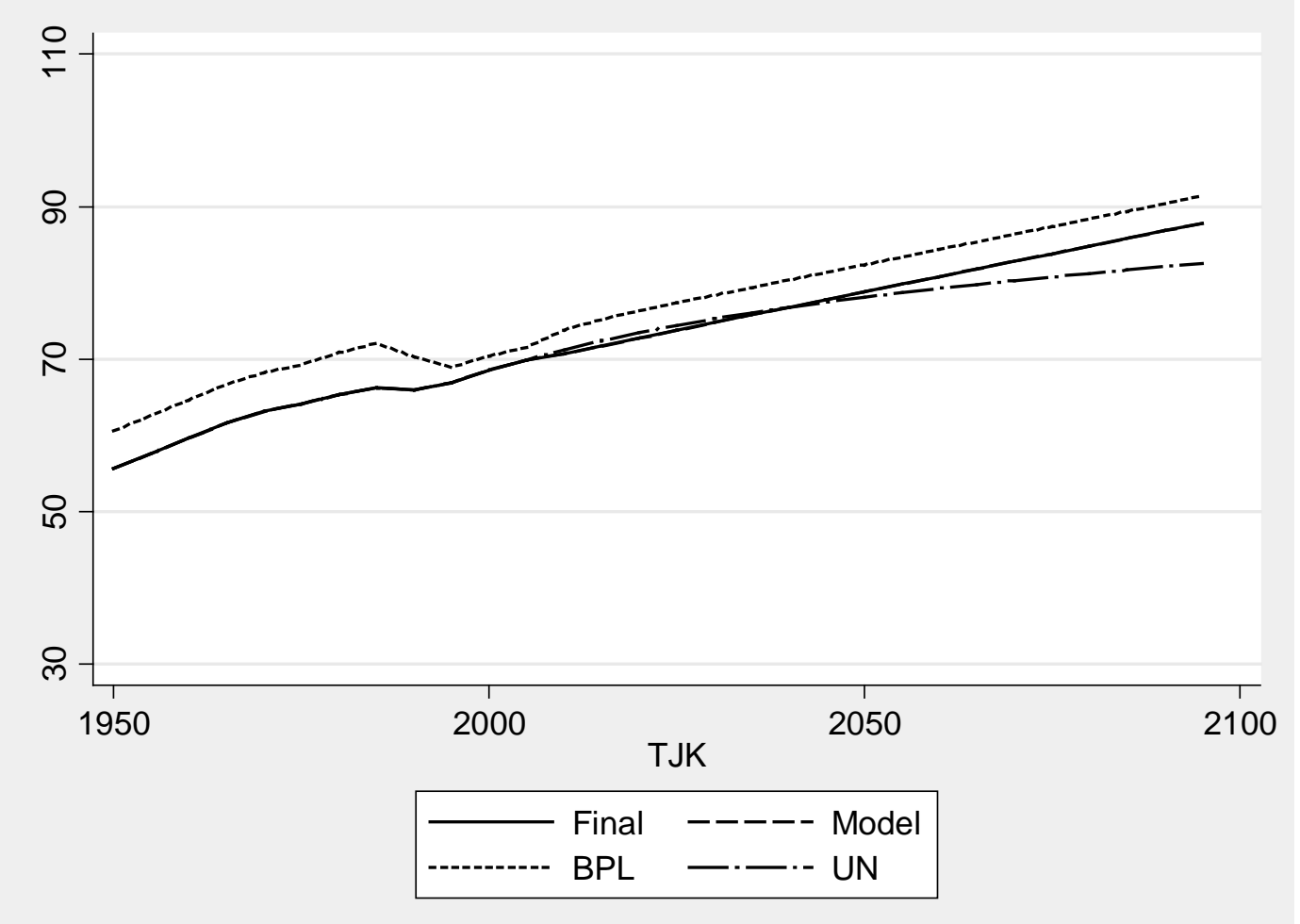

Figure 5. Tajikistan (BPL: Kazakhstan) 


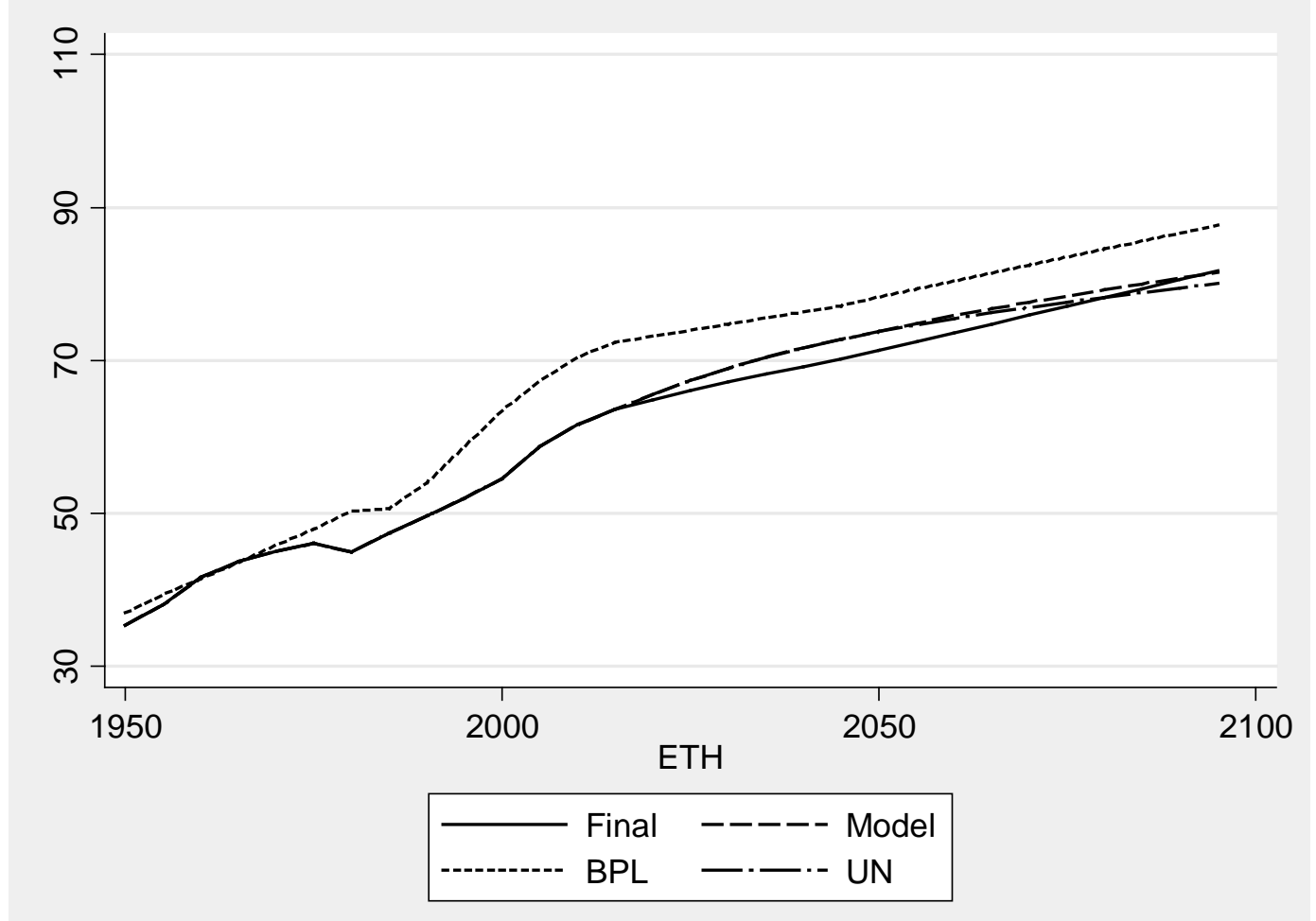

Figure 6. Ethiopia (BPL: Madagascar)

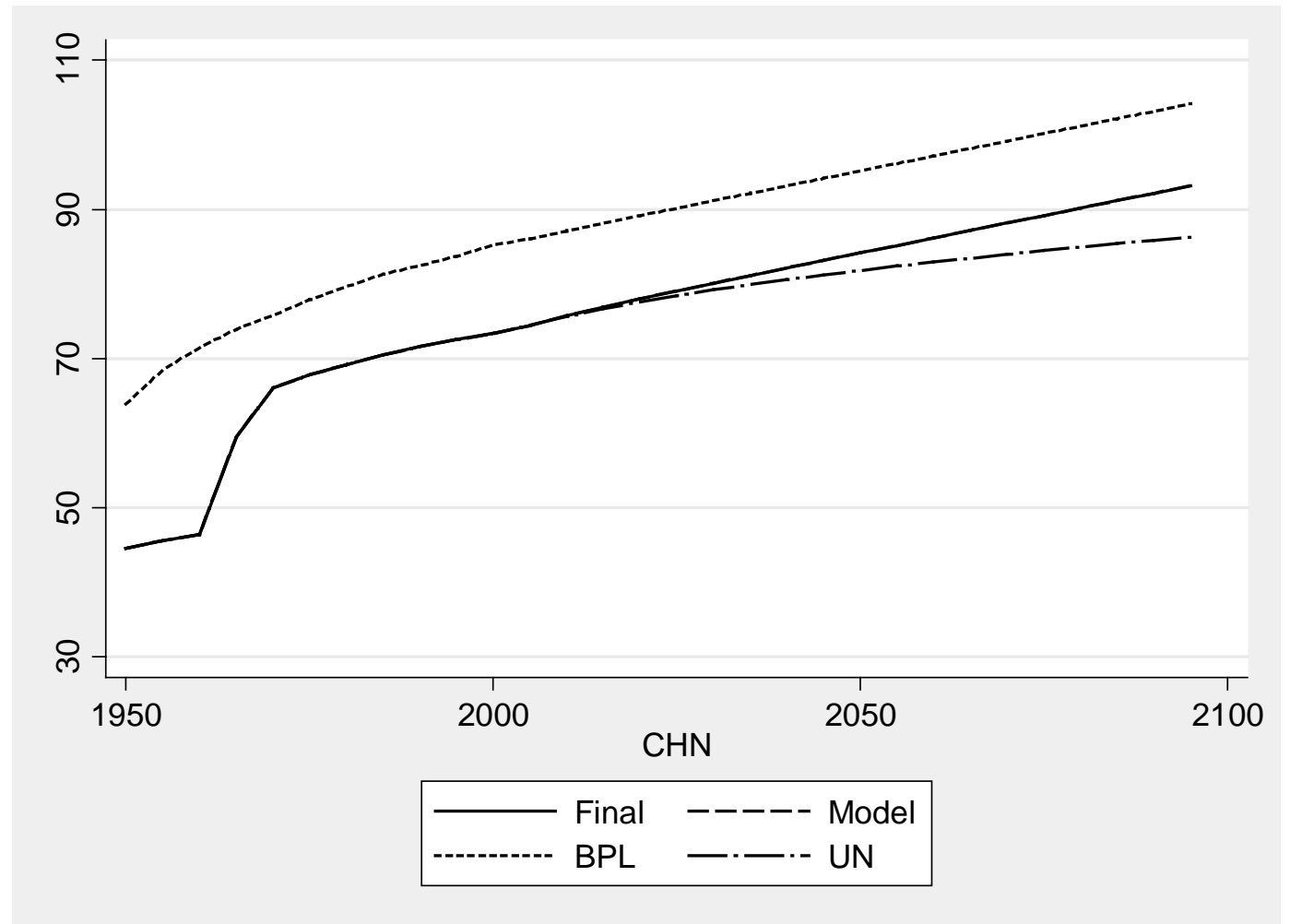

Figure 7. China (BPL: Japan) 


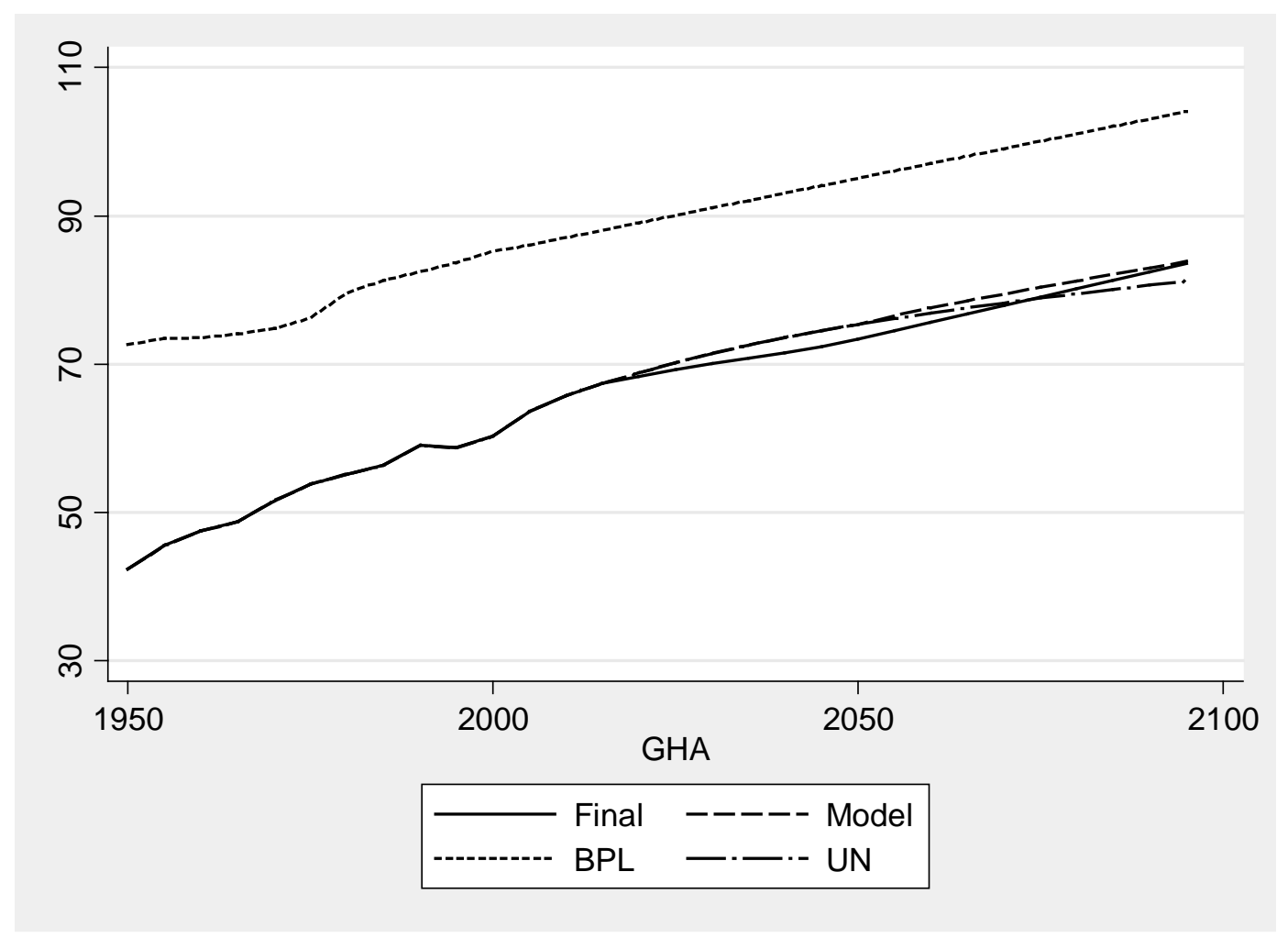

Figure 8. Ghana (BPL: Global forerunner)

\section{Discussion}

We have developed an econometric model that operationalizes the concept of mortality convergence for 159 countries in the world explicitly varying the speed of convergence i.e. taking into account differential rates of linear increase in life expectancy across group of countries. The model is grounded on empirical data and produces life expectancy forecasts which are a function of country-specific stochastic and structural trends in female life expectancy, as well as a function of stochastic and structural differences between forerunners and non-forerunners. Therefore, the model takes into account country-specific and between countries heterogeneity at various levels and it is econometrically unbiased.

For countries with generalized HIV and AIDS epidemics (38 countries), we don't assume convergence for the period 2010 to 2050. For the latter, we endorse the United Nations medium scenario, which explicitly models the impact of HIV and AIDS with an epidemiological model (Alkema et al. 2007; Brown et al. 2010; Raftery and Bao 2010).

Our model incorporates uncertainty through an expert-based approach. To this end, experts were asked to participate and provide an argument-based underpinning of numerical estimates about future trends in life expectancy in high and low mortality countries. Therefore, we develop a framework that operationalizes source-experts and meta-experts opinions and combines their estimates with our life expectancy forecasts. 
The methodology proposed essentially presents a formal framework where extrapolative methods are combined with expert-based argument approaches. In addition, it is based on empirical data, it is replicable, relatively simple and transparent.

\section{References}

Alkema, L., Raftery, A. E. \& Clark, S. J. (2007). Probabilistic projections of HIV prevalence using Bayesian melding. The Annals of Applied Statistics 1(1): 229248.

Anand, S. \& Ravallion, M. (1993). Human development in poor countries: On the role of private incomes and public services. The Journal of Economic Perspectives 7(1): 133-150.

Barro, R. J. \& Sala-I-Martin, X. (1995). Economic Growth. London: Mac Graw Hill.

Bidani, B. \& Ravallion, M. (1997). Decomposing social indicators using distributional data. Journal of Econometrics 77(1): 125-139.

Booth, H. \& Tickle, L. (2008). Mortality modelling and forecasting: A review of methods. Annals of Actuarial Science 3(1/2): 3-43.

Brown, T., Bao, L., Raftery, A. E., Salomon, J. A., Baggaley, R. F., Stover, J. \& Gerland, P. (2010). Modelling HIV epidemics in the antiretroviral era: The UNAIDS estimation and projection package 2009. Sexually Transmitted Infections 86: ii3-ii10.

GHI. (2011). Namibia: Global Health Initiative 2011-2015/16. Accessible online: http://www.ghi.gov/documents/organization/192604.pdf.

Heuveline, P. (1999). The global and regional impact of mortality and fertility transitions, 1950-2000. Population and Development Review 25(4): 681-702.

Hyndman, R. J., Booth, H. \& Yasmeen, F. (2011). Coherent mortality forecasting: the product-ratio method with functional time series models. Monash Econometrics and Business Statistics Working Papers.

Lalic, N. \& Raftery, A. E. (2011). Joint Probabilistic Projection of Female and Male Life Expectancy. San Francisco: Population Association of America.

Landau, R., Taylor, T. \& Wright, G. (1996). The Mosaic of Economic Growth. Standford: Stanford University Press.

Lee, R. (2003). Mortality forecasts and linear life expectancy trends. Social Insurance Studies 3: 19.

Lee, R. D. \& Carter, L. R. (1992). Modeling and forecasting U. S. mortality. Journal of the American Statistical Association 87(419): 659-671.

Lutz, W. \& Goldstein, J. R. (2004). Introduction: How to deal with uncertainty in population forecasting? International Statistical Review 72(1): 1-4.

Lutz, W., Sanderson, W. \& Scherbov, S. (2001). The end of world population growth. Laxenburg: International Institute for Applied Systems Analysis.

Lutz, W., Sanderson, W. C. \& Scherbov, S. (1998). Expert-based probabilistic population projections. Population and Development Review 24: 139-155.

Lutz, W. \& Scherbov, S. (1998). An expert-based framework for probabilistic national population projections: The example of Austria. European Journal of Population/Revue européenne de Démographiy 14(1): 1-17.

McMichael, A. J., McKee, M., Shkolnikov, V. \& Valkonen, T. (2004). Mortality trends and setbacks: global convergence or divergence? The Lancet 363(9415): 11551159. 
Oeppen, J. (2006). Life expectancy convergence among nations since 1820: separating the effects of technology and income. In: T. Bengtsson (ed.) Perspectives on mortality forecasting-III. The linear rise in life expectancy: history and prospects. Försäkringskassan: Swedish Social Insurance Agency, pp. 55-82.

Oeppen, J. \& Vaupel, J. W. (2002). Broken limits to life expectancy. Science 296(5570): 1029-1031.

Omran, A. R. (1971). The epidemiologic transition: A theory of the epidemiology of population change. The Milbank Memorial Fund Quarterly 49(4): 509-538.

Raftery, A. E. \& Bao, L. (2010). Estimating and projecting trends in HIV/AIDS generalized epidemics using incremental mixture importance sampling. Biometrics 66(4): 1162-1173.

Raftery, A. E., Li, N., Ševčíková, H., Gerland, P. \& Heilig, G. K. (2012). Bayesian probabilistic population projections for all countries. Proceedings of the National Academy of Sciences 109(35): 13915-13921.

Ranis, G. \& Stewart, F. (2000). Strategies for success in human development. Journal of Human Development 1: 49-69.

Sanderson, W. \& Scherbov, S. (2004). Putting Oeppen and Vaupel to work: On the road to new stochastic mortality forecasts. IIASA Interim Report IR-04-049. Laxenburg: International Institute for Applied Systems Analysis.

Torri, T. \& Vaupel, J. W. (2012). Forecasting life expectancy in an international context. International Journal of Forecasting 28(2): 519-531.

UNAIDS. (2010). UNAIDS Global Report on the AIDS epidemic. Accessible online: http://www.unaids.org/globalreport/global_report.htm.

United Nations (2011). World Population Prospects: The 2010 Revision. New York.

White, K. M. (2002). Longevity advances in high-income countries, 1955-96. Population and Development Review 28(1): 59-76.

Wilson, C. (2001). On the scale of global demographic convergence 1950-2000. Population and Development Review 27(1): 155-171. 